\title{
Measurement Error of a Simplified Protocol for Quantitative Sensory Tests in Chronic Pain Patients
}

\author{
Monika Müller, MD, *† José Alberto Biurrun Manresa, PhD, + Andreas Limacher, PhD, $\uparrow \$$ \\ Konrad Streitberger, MD, * Peter Jüni, MD,// Ole Koseler Andersen, PhD, $\neq$ and Michele Curatolo, MD, PhD $\neq^{* *}$
}

\begin{abstract}
Background and Objectives: Large-scale application of Quantitative Sensory Tests (QST) is impaired by lacking standardized testing protocols. One unclear methodological aspect is the number of records needed to minimize measurement error. Traditionally, measurements are repeated 3 to 5 times, and their mean value is considered. When transferring QST to a clinical setting, reducing the number of records would be desirable to meet the time constraints encountered in a routine clinical environment and to reduce the testing burden to chronic pain patients. However, there might be a trade-off between measurement error and number of records. We determined the measurement error of a single versus the mean of 3 records of pressure pain detection threshold (PPDT), electrical pain detection threshold (EPDT), and nociceptive withdrawal reflex threshold (NWRT) in 429 chronic pain patients recruited in a routine clinical setting.
\end{abstract}

Methods: We calculated intraclass correlation coefficients and performed a Bland-Altman analysis.

Results: Intraclass correlation coefficients were all clearly greater than 0.75 , and Bland-Altman analysis showed minute systematic errors with small point estimates and narrow $95 \%$ confidence intervals. Reducing the number of records from traditionally 3 to only 1 did not lead to relevant measurement error in PPDT, EPDT, or NWRT.

Conclusions: This study contributes to a standardized QST protocol, and based on the minimal measurement error of 1 single record of PPDT, EPDT, and NWRT, we submit to reduce the testing burden. This would allow saving time, resources, and patient discomfort.

(Reg Anesth Pain Med 2017;42: 660-668)
From the *Department of Anaesthesiology and Pain Medicine, Inselspital, Bern University Hospital, Bern; †Institute of Social and Preventive Medicine, University of Bern, Bern, Switzerland; $\$$ Center for Sensory-Motor Interaction, Department of Health Science and Technology, Aalborg University, Aalborg, Denmark; §Clinical Trials Unit Bern, Department of Clinical Research, University of Bern, Bern, Switzerland; \|Applied Health Research Centre (AHRC) Li Ka Shing Knowledge Institute of St Michael's Hospital, University of Toronto, Toronto, Ontario, Canada; and **Department of Anesthesiology and Pain Medicine, University of Washington, Seattle, WA

Accepted for publication March 20, 2017.

Address correspondence to: Michele Curatolo, MD, PhD, Department of Anesthesiology and Pain Medicine, University of Washington, $1959 \mathrm{NE}$ Pacific St, Box 356540 Seattle, WA 98195 (e-mail: curatolo@uw.edu).

M.M. and J.A.B.M. contributed equally to this work.

Author contributions: M.M., J.A.B.M., and M.C. conceived the study. M.M. and K.S. were responsible for data collection. M.M., J.A.B.M., and A.L. did the data analysis. M.M. and M.C. wrote the first draft of the paper, and all authors contributed to the final draft.

The study was funded by the Scientific Funds of the Department of Anaesthesiology and Pain Medicine, Inselspital, Bern University Hospital.

The results of this study were not presented at any conference; nor was the manuscript under consideration by any other journal.

The authors declare no conflict of interest.

Supplemental digital content is available for this article. Direct URL citations appear in the printed text and are provided in the HTML and PDF versions of this article on the journal's Web site (www.rapm.org).

Copyright (C) 2017 by American Society of Regional Anesthesia and Pain Medicine

ISSN: 1098-7339

DOI: 10.1097/AAP.0000000000000640
C hronic pain is frequently characterized by a discrepancy between objective signs of tissue damage and magnitude of pain and disability. Altered central pain processing may explain part of the complex phenomenology of chronic pain, and thus the paradigm of central hypersensitivity was widely studied during the last decade. ${ }^{1-3}$ The assessment of altered central pain processing using Quantitative Sensory Tests (QSTs) may include the measurement of pain intensity or pain thresholds after the application of painful stimuli and the assessment of the spinal nociceptive withdrawal reflex (NWR) as involuntary response to nociceptive stimulation. ${ }^{4,5}$ There is a large body of evidence that patients with different chronic pain syndromes display signs of altered central pain processing as assessed by QSTs.

Knowledge on the presence and importance of altered central pain processing has led to an increasing application of QSTs in research and clinical practice. ${ }^{3}$ However, wide use of QSTs is impaired by lacking consensus protocols concerning the type of QSTs to be used, the body region of their application, the order of testing modality, and further methodological aspects such as the number of records needed to yield valid test results. ${ }^{3,6}$ To ensure comparability and reproducibility of research results and to facilitate wide use in clinical practice, an evidence-based approach toward standardization of the QST assessment procedure is needed.

One unclear methodological aspect of QST is the number of records needed to balance time for testing, patient discomfort, and measurement error. Traditionally, tests are repeated 3 to 5 times and their mean value is considered as test result. ${ }^{3,6,7}$ This is done based on the assumption that single records would be potentially inaccurate, and multiple records would compensate for potential outlier results. When transferring QSTs to a clinical setting, reducing the number of records would be desirable to meet the time constraints commonly encountered in a routine clinical environment and to reduce the testing burden to chronic pain patients. However, most likely there will always be a trade-off between measurement error and number of records. To contribute to a more standardized QST assessment procedure while accounting for different requirements in a routine clinical environment, we conducted a large-scale method comparison analysis. We determined the measurement error of a single versus the mean of 3 records of pressure pain detection threshold (PPDT), electrical pain detection threshold (EPDT), and NWR threshold (NWRT) in chronic pain patients referred to a tertiary care facility.

\section{METHODS}

\section{Participants}

In 2011, we included QST as part of a routine multimodal patient assessment at our tertiary care outpatient's facility. The present analysis was performed in a subsample of participants in whom we estimated the prevalence of central hypersensitivity as assessed by QSTs. ${ }^{8}$ All patients who suffered from pain lasting more than 3 months and were referred between July 1, 2011, and June 30,2012, to the Department of Anaesthesiology and 
Pain Medicine of the University Hospital of Bern in Switzerland were eligible for the study. We excluded patients with neurological comorbidities potentially affecting the neurological function of the lower extremity to be tested (palsy, paresthesia, polyneuropathy), patients with rheumatic inflammatory disease, and patients with chronic pain as result of evident peripheral lesions (oncological pain in the region of infiltration by a primary tumor, metastasis, or peripheral vascular disorder). Other reasons of exclusion were psychiatric comorbidity except unipolar depressive disorder, pregnancy, and language problems. We informed all patients in written form on the background of the QSTs and on the use of their data for scientific purposes. All patients gave informed consent before the tests, which were performed according to a prospective protocol approved by the local research ethics committee and in accordance with the Declaration of the World Medical Association. ${ }^{9}$

\section{Sociodemographic, Psychological, and Clinical Characteristics}

We recorded sociodemographic, psychological, and clinical characteristics for descriptive purposes. Sociodemographic characteristics were sex, age, and working status. Psychological characteristics were depression and catastrophizing assessed with the Fast Screen Beck Depression Inventory (BDI-FS) ${ }^{10}$ and the Catastrophizing Scale of the Coping Strategies Questionnaire (CSQ). ${ }^{11}$ Clinical characteristics were body mass index and pain-related variables such as history of trauma or surgery related to pain, pain duration, pain intensity, pain-related life interference, type of pain, and current pain medication. We measured pain intensity and pain-related sleep interference with a numerical rating scales (NRS) ranging from 0 (no pain or interference) to 10 (worst pain or interference imaginable). We classified the type of pain as musculoskeletal, neuropathic, orofacial, or visceral and summarized pain syndromes rarely encountered in our clinic such as noncervicogenic headache, complex regional pain syndrome, and phantom limb pain in the separate class of "rare pain syndromes." We based the definition of the type of pain on our previous publication of the prevalence of central hypersensitivity as assessed by QSTs. ${ }^{8}$ We defined daily intake of pain medication as intake of at least 1 pain medication of the following classes: opioids, nonsteroidal anti-inflammatory drugs, acetaminophen, metamizole, or analgesic comedication such as antidepressants and/or anticonvulsants.

\section{Quantitative Sensory Testing}

We implemented the assessment of PPDT at the second toe, pain detection threshold after single electrical stimulation (EPDT), and the NWRT after single electrical stimulation because these assessments can be easily applied in routine clinical practice and in a previous investigation displayed good discriminative ability for hypersensitivity among 26 tests. ${ }^{12}$ Four different health care professionals routinely performed all QSTs at the extremity contralateral to the side of most pain. In case of bilateral pain, the testing extremity was randomly selected according to a computer-generated list. The assessment was standardized according to a prespecified protocol and included standardized oral explanation of the experimental setting, training session, and recording phase for all patients. The training session is considered essential to familiarize patients with the stimulation procedure. ${ }^{6}$ These sessions took on average 5 minutes and included 3 training records for pressure stimulation to assess PPDT and 3 training records for electrical stimulation to assess EPDT and NWRT. Thereafter we again performed 3 records to definitively assess PPDT, EPDT, and NWRT. We measured pain detection threshold after pressure stimulation at the center of the pulp of the second toe using an electronic pressure algometer with a $1 \mathrm{~cm}^{2}$ surface probe (Somedic AB, Sösdala,
Sweden). ${ }^{13}$ Pressure was increased at a rate of $30 \mathrm{kPa} / \mathrm{s}$ until patients perceived the stimulus as painful, what we defined as pain detection threshold. In case the stimulation was not perceived as painful, we considered the maximum stimulation intensity of $1000 \mathrm{kPa}$ as threshold. We performed electrical stimulation of the sural nerve with bipolar surface $\mathrm{Ag} / \mathrm{AgCl}$ electrodes placed distal to the lateral malleolus. A computer-controlled constant current stimulator (NCS System, Evidence 3102 evo; Neurosoft, Moscow, Russia) delivered a train-of-5 1-millisecond square-wave pulses of an overall duration of 25 milliseconds, which was perceived as a single stimulus by the patients. In a single increasing intensity staircase, the current intensity was increased from $1 \mathrm{~mA}$ in steps of $1 \mathrm{~mA}$ until the electrical stimulus was perceived as painful (EPDT), and until an NWR of the biceps femoris with an amplitude higher than $20 \mu \mathrm{V}$ for at least 10 milliseconds in the 50 - to 150-millisecond postestimation interval was elicited (NWRT). ${ }^{14,15}$ We did not apply a maximum current intensity.

\section{Statistical Analysis}

Quantitative Sensory Tests were not performed or not repeated 3 times for logistic reasons in 103 patients (Fig. 1). We excluded these patients from the method comparison analysis but evaluated a potential selection bias by comparing their sociodemographic, psychological, and clinical characteristics, with patients included in the analysis using $\chi^{2}$ and Student $t$ tests. We also compared characteristics of patients with and without successful NWRT assessment using $\chi^{2}$ and Student $t$ test. To evaluate measurement error when reducing the number of records to 1 , we first calculated intraclass correlation coefficients (ICCs) based on mixed-effects linear regression models with a random intercept for subjects to account for clustering of repeated records within patients. The ICC was calculated as the estimated variance of the measurements between subjects divided by the sum of the estimated variances of the measurements between and within subjects. Then, we performed a method comparison analysis as suggested by Bland and Altman. ${ }^{16-18}$ We considered the value of record 1 as measurement method M2 and compared it with the mean value of records 1,2 , and 3 , which was considered as measurement method M1.

In a first set of mixed-effects linear regression models, we used the 3 single records of PPDT, EPDT, and NWRT as dependent variable and the subject identifier as random effect. Intraclass correlation coefficients in these models estimate the concordance of the 3 records and show how much of the total variance can be attributed to within-patient variability and between-patient variability. We then included an indicator variable for record number to investigate a possible systematic effect of sequential recording.

In a second set of mixed-effects linear regression models, we used measurement methods (M2 vs M1) of PPDT, EPDT, and NWRT as dependent variable and the subject identifier as random effect. The ICCs in these models estimate the concordance between the 2 measurement methods, thus representing the measurement error relative to the variability between patients. Intraclass correlation coefficient values greater than 0.75 suggest an excellent, between 0.4 and 0.75 a moderate, and less than 0.4 a poor correlation of the 3 records or of the 2 measurement methods. ${ }^{19}$ Then, we performed a Bland-Altman analysis. ${ }^{16-18}$ Bland and Altman suggested that the extent of agreement between 2 measurement methods could be examined by comparing the differences between the pairs of measurements with the mean of each pair. ${ }^{16-18}$ The mean difference between the 2 methods explains whether there is a systematic error in the new method (M2) as compared with the standard method (M1). The limits of agreement are defined as the systematic error (or mean difference) \pm 1.96 times 


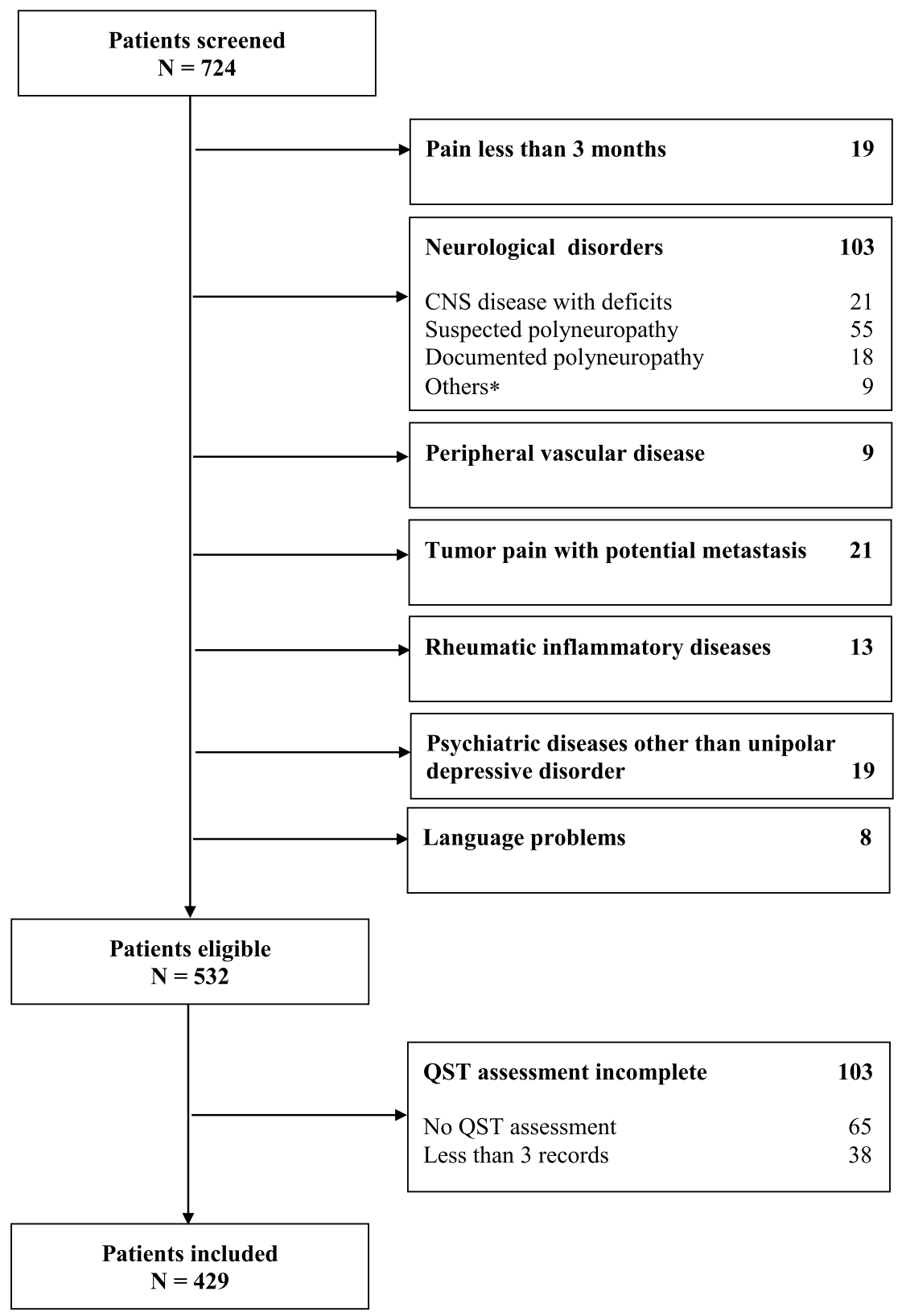

FIGURE 1. Flowchart of patients undergoing first consultation between July 1, 2011, and June 30, 2012. *Two patients with neuroborreliosis, 2 patients with bilateral paresis of unknown origin, 3 patients with bilateral sensibility disorders of unknown origin, and 2 patients with restless legs syndrome.

the SD of the difference. They delimit the range within which $95 \%$ of the differences between results of QSTs may be expected to lie if the number of records is limited to 1 . In close relation to this definition, the coefficient of repeatability (CR) is defined as the value below which $95 \%$ of the absolute differences between thresholds may be expected to lie. If the systematic error is close to 0 , the limits of agreement and the $\mathrm{CR}$ are expected to be similar. We generated Bland-Altman plots allowing visual inspection of the measurement error by plotting the mean of the 2 measurement methods against the difference of the 2 methods. If the measurement error is unrelated to the size of the outcome variable, a random scatter can be expected.
Finally, we conducted 2 sensitivity analyses and several stratified exploratory analyses. First, we changed the definition of measurement method M1 to account for the high arithmetic correlation between the mean value of records 1,2 , and 3 and the value of record 1 . We thus compared the value of record 1 (M2) with the mean value of records 2 and 3 (M1). Second, we stratified the analysis according to assessor to evaluate if measurement error was comparable in all 4 assessors. To evaluate the effect of gender (male vs female), age ( $\geq 65$ vs $<65$ years), depression (BDI-FS $\geq 4$ vs $<4$ ), catastrophizing (CSQ median value of $\geq 3.17$ vs $<3.17$ ), pain duration (below 1 year, $1-2$ years, $>2$ years), type of pain, pain intensity (NRS median value of $\geq 6$ vs $<6$ ), daily 
intake of any medication (yes vs no), and daily intake of different classes of pain medications (yes vs no) on measurement error, we performed secondary exploratory analyses stratified according to these variables (Appendix, Supplemental Digital Content 1, http:// links.lww.com/AAP/A210). We performed all statistical analyses with STATA (version 12.1; StataCorp, College Station, Texas).

\section{RESULTS}

We screened 724 patients who were referred to our pain clinic for a first consultation between July 1, 2011, and June 30, 2012, and excluded 192 patients (Fig. 1). The most important reason for exclusion was concomitant neurological disorder, accounting for $54 \%$ of all exclusions. For logistic reasons, most commonly due to time constraints, we did not perform any QST in 65 patients $(12 \%)$ and were unable to repeat QST 3 times in 38 patients $(7 \%)$ of all included patients. These 103 patients with incomplete QSTs were significantly older $(P=0.002)$ as compared with the 429 patients analyzed, but did not differ in any other patients' characteristics listed in Table 1. Data on PPDT and EPDT were complete. Maximum PPDTs for records 1, 2, and 3 were 982, 736, and $707 \mathrm{kPa}$, respectively. We were unable to determine NWRT in 130 patients because electrical stimulation became intolerable before a reflex could be detected. We found no differences in characteristics of patients with and without successful NWRT assessment using $\chi^{2}$ and Student $t$ test and, except for age and daily intake of any pain medication. Mean ages were 48.6 (SD, 14.8)years and 52.5 (SD, 14.5)years in patients with and without NWRT, respectively $(P=0.01)$. A smaller proportion of patients with NWRT took any pain medication $(69 \%$ vs $80 \%, P=0.02)$. Intraclass correlation coefficients and results of the BlandAltman analysis were based on 429 patients for PPDT and EPDT and on a subsample of 299 patients for NWRT.

Table 1 shows sociodemographic, psychological, and clinical characteristics of all 429 patients. More than half were female $(56 \%)$, suffered from depression (54\%), and had pain lasting more than 2 years $(58 \%)$. One hundred forty-seven patients $(33 \%)$ were unemployed because of their chronic pain condition. Most frequently, patients suffered from musculoskeletal pain (287 patients $[67 \%])$. Three hundred ten patients $(72 \%)$ took at least 1 pain medication on a daily basis. One hundred eleven $(26 \%)$ of all patients regularly took opioids; $126(29 \%)$, nonsteroidal anti-inflammatory drugs; 44 (10\%), metamizole; 150 (35\%), acetaminophen; and $128(30 \%)$ took coanalgesics such as antidepressants or anticonvulsants. Figure 2 illustrates results of PPDT, EPDT, and NWRT for records 1,2, and 3. The mean values and SDs were similar for all 3 records. We found evidence for a systematic effect of sequential recording for PPDT and EPDT but not for NWRT. Values of PPDT significantly increased, and values for EPDT significantly decreased for every additional record. The ICCs of the 3 records were 0.91 (95\% confidence interval [CI], 0.89-0.92), 0.95 (95\% CI, 0.94-0.95), and 0.90 (95\% CI, 0.88-0.91) for PPDT, EPDT, and NWRT, respectively.

Table 2 shows the results of the main analysis comparing record 1 with the mean of all 3 records, as well as the sensitivity analysis comparing record 1 with the mean of records 2 and 3 for all 3 QSTs. As for the main analysis, the point estimates of the ICCs were $0.96,0.97$, and 0.95 for PPDT, EPDT, and NWRT, respectively, with lower bounds of the $95 \%$ CIs greater than 0.93 . The Bland-Atman analysis showed a slight overestimation for PPDT if the number of records is limited to one, as compared with the mean of 3 records, with a systematic error of $5.98 \mathrm{kPa}(95 \% \mathrm{CI}$, 2.81-9.14 kPa). For EPDT, results were slightly underestimated when reducing the number of records to 1 . For NWRT, again the point estimate indicated that results were underestimated when
TABLE 1. Sociodemographic, Psychological, and Clinical Characteristics of 429 Chronic Pain Patients

\begin{tabular}{lc}
\hline & $\begin{array}{c}\text { Mean (SD) or No. } \\
\text { of Patients (\%) }\end{array}$ \\
\hline Sociodemographic characteristics & \\
Females & $239(56 \%)$ \\
Age, y & $49.8(14.6)$ \\
Working status & $131(31 \%)$ \\
$\quad$ Regular work as usual & \\
Reduced work due to pain & $73(17 \%)$ \\
No work & $137(33 \%)$ \\
Retired or studying & $76(18 \%)$ \\
Psychological characteristics & \\
Depression (BDI-FS, ${ }^{*}$ cutoff $\left.\geq 4\right)$ & $221(54 \%)$ \\
Catastrophizing (CSQ $\dagger)$ & $3.2(1.4)$ \\
Clinical characteristics & \\
Body mass index, kg/m ${ }^{2}$ & $26.4(5.3)$ \\
Pain duration & \\
$\quad<1$ y & $98(24 \%)$ \\
1-2 y & $75(18 \%)$ \\
>2 y & $238(58 \%)$ \\
Type of pain & \\
Musculoskeletal & \\
Neuropathic & $287(67 \%)$ \\
Orofacial & $55(13 \%)$ \\
Visceral/urogenital & $34(8 \%)$ \\
Rare pain syndromes & $30(7 \%)$ \\
Maxients with bilateral pain & $23(5 \%)$ \\
Minimum pain in the last $24 \mathrm{~h}(\mathrm{NRS} \ddagger)$ & $167(39 \%)$ \\
Average pain in the last $24 \mathrm{~h}(\mathrm{NRS} \ddagger)$ & $7.2(2.2)$ \\
Dain-related sleep inferences (NRS $\ddagger)$ & $4.2(2.6)$ \\
\hline Vaily intake of at least 1 pain medication & $6.2(2.2)$ \\
& $5.3(3.0)$ \\
& $310(72 \%)$ \\
\hline
\end{tabular}

Values are numbers (percentages) or means (SDs).

*BDI-FS from 0 (no depression) to 21 (maximum depression).

$\uparrow \mathrm{CSQ}$ from 0 (no catastrophizing) to 6 (maximum catastrophizing).

$\$ N R S$ from 0 (no pain/no interference) and 10 (maximum pain/ maximum interference).

reducing the number of records to 1 ; however, the $95 \%$ CI was compatible with both underestimation and overestimation (systematic error of $-0.11 \mathrm{~mA} ; 95 \% \mathrm{CI},-0.26-0.05 \mathrm{~mA}$ ). The CRs were $66.7 \mathrm{kPa}, 1.44 \mathrm{~mA}$, and $2.75 \mathrm{~mA}$ for PPDT, EPDT, and NWRT, respectively. As for the sensitivity analysis, ICCs were robust for all 3 QSTs. Measurement errors were slightly larger than in the main analysis with systematic errors of $8.72 \mathrm{kPa},-0.55 \mathrm{~mA}$, and $-0.36 \mathrm{~mA}$, as well as CRs of $100 \mathrm{kPa}, 2.31 \mathrm{~mA}$, and $4.19 \mathrm{~mA}$ for PPDT, EPDT, and NWRT, respectively. Figure 3 illustrates Bland-Altman plots of PPDT, EPDT, and NWRT of the main and the sensitivity analysis for visual inspection of the maximal measurement error.

Table 3 displays the results of the second sensitivity analysis investigating measurement error after stratifying for assessor. Again, point estimates and lower bounds of $95 \%$ CI of all ICCs were clearly greater than 0.75 , and ICCs per testing modality were comparable between assessors. The Bland-Altman analysis showed numerically different systematic errors between the 4 assessors for 


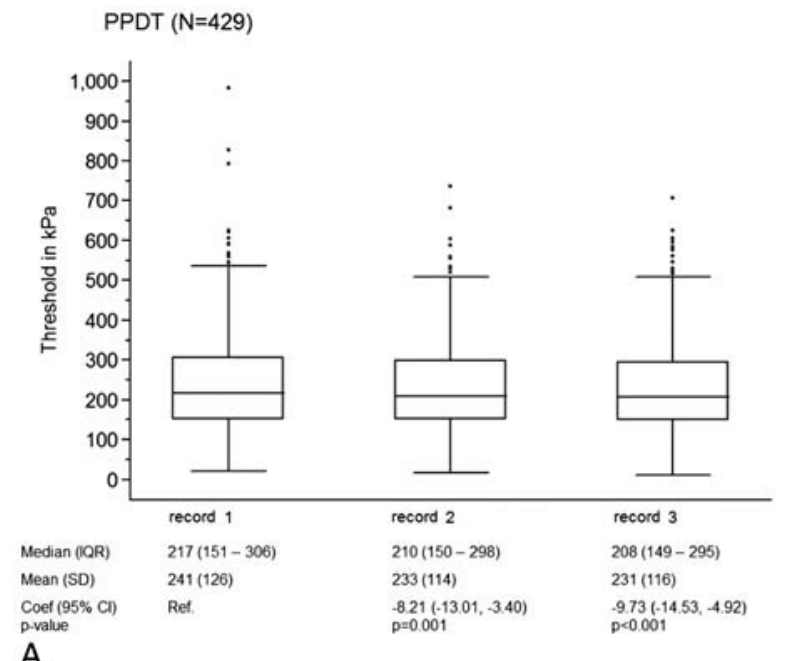

A

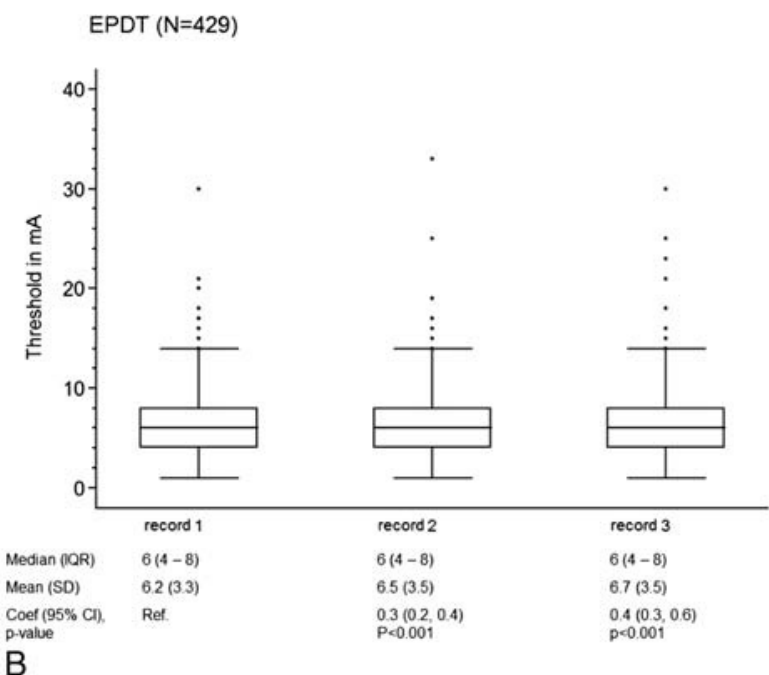

B

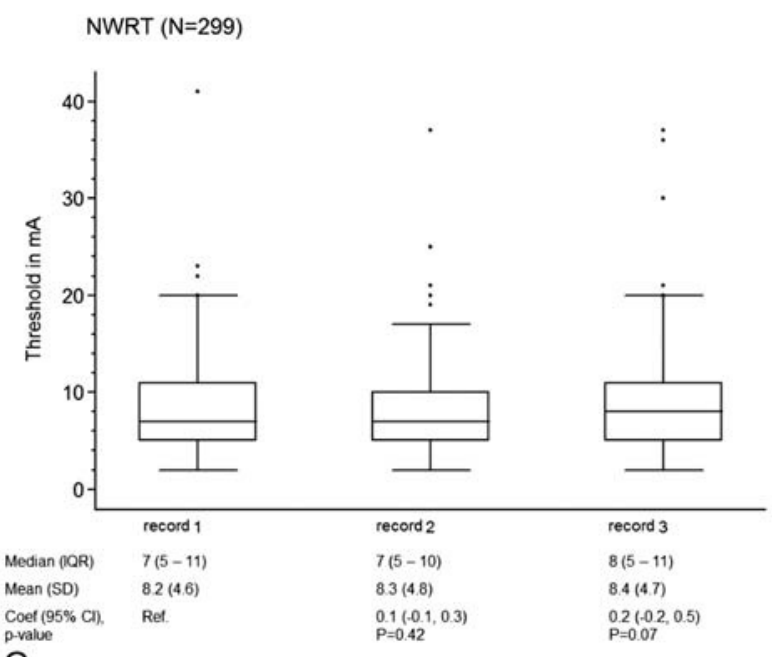

C

FIGURE 2. Box plots of each record of (A) PPDT, (B) EPDT, and (C) NWRT after single electrical stimulation. Values are medians with interquartile range (IQR), means with SDs, and $\beta$ coefficients (Coef) with corresponding $95 \% \mathrm{Cls}$ and $P$ values based on mixed-effects linear regressions. all 3 tests, with PPDT being the test with the largest difference between assessor on the one hand and the largest discrepancy from the main analysis on the other hand. For EPDT and NWRT, however, the differences between assessors were small, and the point estimates per assessor were comparable to the results of the main analysis. Results of all secondary exploratory analyses stratified according to gender, age, depression, degree of catastrophizing, pain duration, type of pain, pain intensity, and daily intake of medication were comparable to the results of the main analysis.

\section{DISCUSSION}

In this large-scale-method comparison analysis of 429 chronic pain patients recruited in a routine clinical setting, we found that reducing the number of records from traditional ${ }^{3,6,7}$ to only 1 did not lead to relevant measurement error in PPDT, EPDT, and NWRT. Point estimates and lower bounds of 95\% CIs of ICCs were clearly greater than 0.75 for all QSTs and thus showed excellent correlation of the 3 sequential records. When directly comparing the value of record 1 with the mean value of records 1,2 , and 3 , ICCs of the 2 measurement methods were 0.95 or higher, again suggesting excellent correlation of both measurement methods. Results of the Bland-Altman analysis showed minute systematic errors with small point estimates and narrow 95\% CIs for all 3 QSTs. The CRs were $66.7 \mathrm{kPa}, 1.44 \mathrm{~mA}$, and $2.75 \mathrm{~mA}$ for PPDT, EPDT, and NWRT, respectively. The low measurement error is evident when observing the narrow lower and upper limits of agreement (Fig. 3), as compared with the corresponding wide ranges of measurement reported in Figure 2. For EPDT, most of the differences between a single measurement and the average 3 will be smaller than $1.7 \mathrm{~mA}$, which corresponds to a variability of less than 2 current steps (usually of $1 \mathrm{~mA}$ ) of the electrical stimulator. For PPDT, most of the differences will be smaller than $70 \mathrm{kPa}$, which corresponds to a variability of approximately 2 seconds on the algometer pressure test (at a rate of $30 \mathrm{kPa} / \mathrm{s}$ ). Results of the sensitivity analysis showed slightly larger measurement error when comparing the value of record 1 with the mean value of records 2 and 3 . The exploratory secondary analyses suggest that there are no relevant effects of gender, age, depression, catastrophizing, pain duration, type of pain, pain intensity, and daily intake of different medications on measurement error. However, the results have to be interpreted with caution because we performed these analyses post hoc, and stratification partly resulted in small subgroups.

To our knowledge, only 1 study assessed measurement error when reducing the number of records to 1 ; the investigation was limited to NWRT. ${ }^{15}$ The authors found high correlations between NWRT of 1 record and mean NWRT of 2 records and concluded that performing only 1 record is acceptable. The findings of our study confirm these results and showed that this was also the case for PPDT and EPDT. Although there are several limitations when using correlation coefficients to estimate measurement error, the authors refrained to perform a formal method comparison analysis in accordance with the method suggested by Bland and Altman. This is the most recommended and commonly used statistical method to estimate measurement error. ${ }^{16-18}$ We therefore interpret our results in the context of previous test-retest reliability studies investigating whether QSTs reliably yield similar results if repeated at different time points. ${ }^{20-22}$ This includes 2 studies that determined measurement error over time for PPDT, ${ }^{21,22} 2$ studies for EPDT, $^{20,22}$ and 1 study for NWRT. ${ }^{20}$ Only our previous studies ${ }^{20,22}$ used Bland-Altman analysis to estimate measurement error. In our first study, we compared QSTs of 3 sessions with a mean of 7.7 days between the sessions. We found mean CRs of the 3 
TABLE 2. Results of the Method Comparison Analysis for 3 Quantitative Sensory Tests Showing Between- and Within-Subject SDs and ICCs Based on Mixed-Effects Linear Regression Models and Systematic Error (SE) and Limits of Agreement (LoA) Based on Bland-Altman Analysis

\begin{tabular}{|c|c|c|c|c|c|c|}
\hline & $\begin{array}{l}\text { SD Between } \\
(95 \% \mathrm{CI})\end{array}$ & $\begin{array}{l}\text { SD Within } \\
(95 \% \text { CI })\end{array}$ & ICC $(95 \%$ CI $)$ & $\mathrm{SE}^{*}(95 \% \mathrm{CI})$ & $\begin{array}{l}\text { Upper LoA } \dagger \\
(95 \% \text { CI) }\end{array}$ & $\begin{array}{l}\text { Lower LoA } \dagger \\
(95 \% \text { CI })\end{array}$ \\
\hline $\begin{array}{l}\text { PPDT, } \$ \\
\mathrm{kPa}\end{array}$ & $118(110-127)$ & $24(22-26)$ & $0.96(0.95-0.97)$ & $5.98(2.81-9.14)$ & $72.66(67.15-78.16)$ & $-60.70(-66.21$ to -55.20$)$ \\
\hline $\begin{array}{c}\text { EPDT, } \S \\
\mathrm{mA}\end{array}$ & $3.28(3.06-3.51)$ & $0.59(0.55-0.63)$ & $0.97(0.96-0.97)$ & $-0.24(-0.31$ to -0.17$)$ & $1.21(1.09-1.33)$ & $-1.68(-1.80$ to -1.56$)$ \\
\hline $\begin{array}{c}\text { NWRT,\| } \\
\text { mA }\end{array}$ & $4.49(4.14-4.88)$ & $0.98(0.91-1.07)$ & $0.95(0.94-0.96)$ & $-0.11(-0.26$ to 0.05$)$ & $2.64(2.37-2.92)$ & $-2.86(-3.13$ to -2.58$)$ \\
\hline \multicolumn{7}{|c|}{ Sensitivity Analysis: Value of Record 1 (M2) vs Mean Value of Records 2 and 3 (M1) } \\
\hline & $\begin{array}{l}\text { SD Between } \\
(95 \% \text { CI })\end{array}$ & $\begin{array}{l}\text { SD Within } \\
\text { (95\% CI) }\end{array}$ & ICC $(95 \% \mathrm{CI})$ & $\mathrm{SE}^{*}(95 \% \mathrm{CI})$ & $\begin{array}{l}\text { Upper LoA }{ }^{\text {Up }} \dagger \\
(95 \% \text { CI) }\end{array}$ & $\begin{array}{l}\text { Lower LoA } \dagger \\
(95 \% \text { CI) }\end{array}$ \\
\hline $\begin{array}{l}\mathrm{PPDT}+\$ \\
\mathrm{kPa}\end{array}$ & $114(106-122)$ & $36(34-38)$ & $0.91(0.89-0.93)$ & $8.72(3.98-13.47)$ & $108.72(100.46-116.97)$ & $-91.28(-99.53$ to -83.02$)$ \\
\hline $\begin{array}{l}\text { EPDT, } \S \\
\text { mA }\end{array}$ & $3.28(3.07-3.51)$ & $0.54(0.50-0.57)$ & $0.97(0.97-0.98)$ & $-0.55(-0.66$ to -0.44$)$ & $1.76(1.57-1.95)$ & $-2.86(-3.05$ to -2.67$)$ \\
\hline $\begin{array}{l}\text { NWRT, } \| \\
\text { mA }\end{array}$ & $4.49(4.14-4.88)$ & $0.98(0.91-1.07)$ & $0.95(0.94-0.96)$ & $-0.36(-0.60$ to -0.12$)$ & $3.84(3.42-4.25)$ & $-4.55(-4.97$ to -4.14$)$ \\
\hline $\begin{array}{l}\text { All val } \\
* \text { SE th } \\
\dagger \text { Lower } \\
\text { †PPDT } \\
\text { §EPDT } \\
\text { |NWR }\end{array}$ & $\begin{array}{l}\text { ues are presented } \mathrm{w} \\
\text { at corresponds to } \mathrm{m} \\
\mathrm{r} \text { and upper limits o } \\
\text { second toe: PPDT } \\
\text { T after single electric } \\
\mathrm{T} \text { after single electri }\end{array}$ & $\begin{array}{l}\text { ith corresponding } 95 \\
\text { lean difference betwe } \\
\text { f agreement that cor } \\
\text { at the second toe ( } \mathrm{n} \\
\text { cal stimulation }(\mathrm{n}= \\
\text { ical stimulation }(\mathrm{n}=\end{array}$ & $\begin{array}{l}5 \% \text { CIs. } \\
\text { een M1 and M2. } \\
\text { respond to SE } \pm 1.9 \\
=429 \text { ). } \\
429 \text { ). } \\
299) \text {. }\end{array}$ & 6 SDs of the SE. & & \\
\hline
\end{tabular}

sessions of 2.3 and $5 \mathrm{~mA}$ for EPDT and NWRT, respectively. ${ }^{20}$ In our second study, we recorded QSTs 3 times within 1 session and calculated the CR for all 3 records to assess within-session reliability. We found a mean CR for all 3 records of $1.3 \mathrm{~mA}$ for EPDT and mean CR of $90 \mathrm{kPa}$ for PPDT, ${ }^{22}$ respectively. Taking ICCs greater than 0.75 into account, the reliability and thus measurement error were concluded acceptable in both studies. ${ }^{20,22} \mathrm{We}$ could confirm our previous findings in this study. We thus submit measurement error to be small when reducing the number of records to 1. Although we found evidence for a systematic effect of sequential recording, the overall correlation of the 3 records was still excellent, with ICCs for the 3 records equal to or greater than 0.90 . This systematic effect is likely the result of sensitization or habituation, although the quantitative changes were minimal. We believe that by reducing the number of records from 3 to 1 we would also be able to avoid such systematic effects as result of sequential recording. Of note, we performed a short training session to familiarize patients with the stimulation procedure before registering the 3 records. This is considered essential before formal testing is started and thus is common practice of QST testing protocols ${ }^{6}$ as it was in the test-retest reliability studies. ${ }^{20-22}$ The ideal clinical QST protocol would require very little to no familiarization to further save time, resources, and patient discomfort. Future research should therefore aim at randomizing patients to different familiarization procedures to evaluate the effect of familiarization on measurement error.

Up-to-date recommendations about the number of records necessary to yield a valid test result were not based on evidence but on expert opinion., ${ }^{3,6,7}$ This is the first large-scale method comparison analysis in chronic pain patients to formally assess the trade-off between measurement error and number of records needed to yield a valid QST result. Strengths of our study include the large sample size with high associated statistical precision and the study setting. We recruited all patients in a routine clinical environment, which suggests generalizability of our results. The measurement error is likely to be even smaller in a highly controlled experimental research setting. In $20 \%$ of all eligible patients, we were unable to perform a complete QST assessment because of logistical reasons, most commonly time constraints in the clinical setting. To evaluate selection bias, we compared these patients with the included patients and found no differences in patients' characteristics except for age. Therefore, results are unlikely to be invalidated by selection bias. The results are contingent on our selection of QST and not necessarily applicable to other QST modalities. However, the results were consistent across 2 different stimulation modalities (pressure and electrical) and 2 different response modalities (pain and reflex thresholds). Our reliability analysis was done within the same session (ie, addressed "internal consistency"). Internal consistency does not necessarily imply "stability over time" (ie, reliability over weeks or months), which may be the target of future research. One limitation was that patients were not randomly allocated to the 4 assessors performing the QSTs. To address this limitation, we performed a sensitivity analysis stratified for assessor. We found different measurement errors for the 4 assessors with largest discrepancies for PPDT and small differences for EPDT and NWRT. As the allocation to the assessor was not randomized, no firm conclusions can be drawn from this finding. Pressure algometry relies on manual testing, which may introduce uncontrolled variability, as opposed to electrical stimulation. The findings suggest that trial sessions 

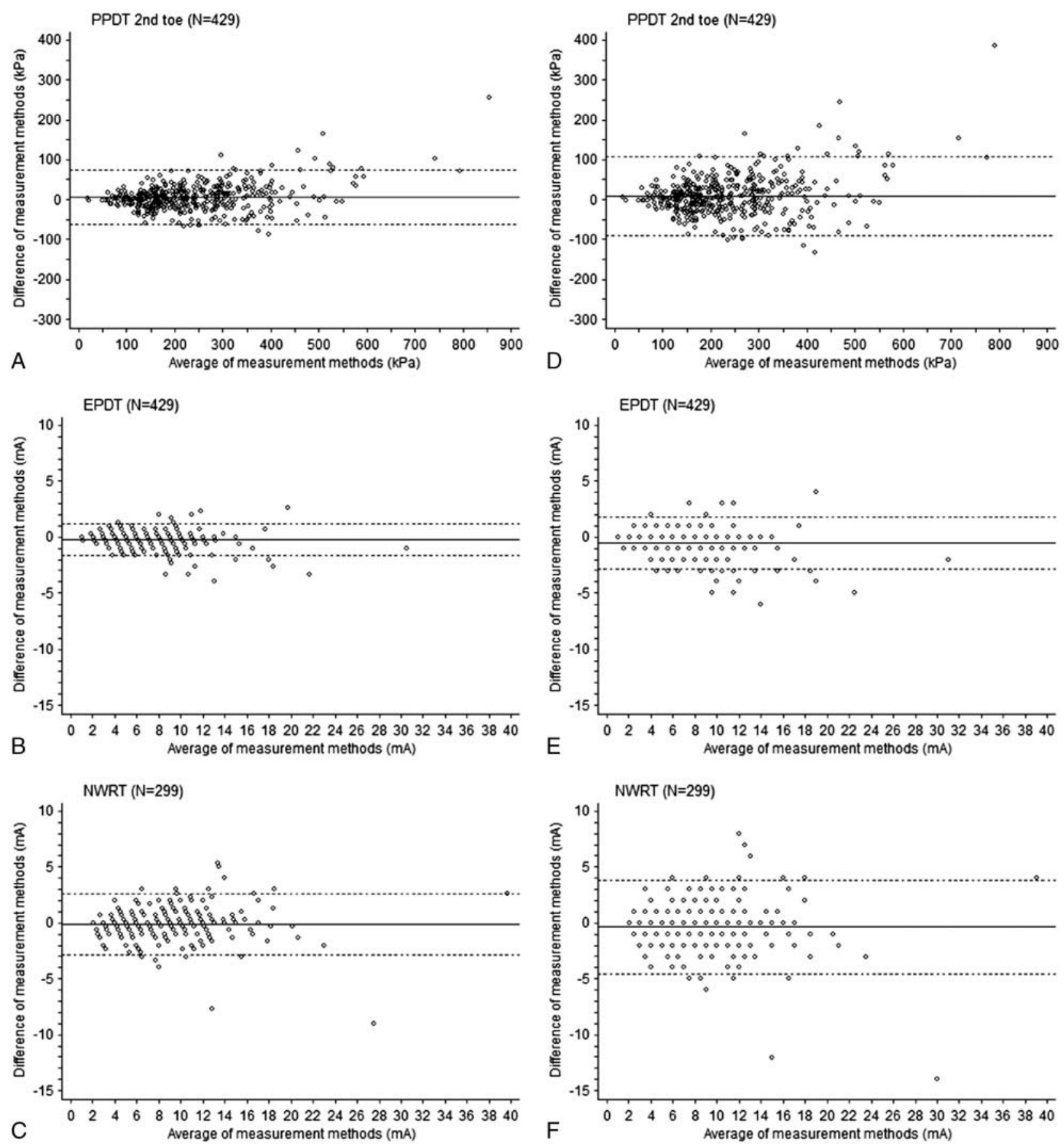

FIGURE 3. Bland-Altman plots of PPDT, EPDT, and NWRT after single electrical stimulation. Solid lines denote systematic error (SE); dashed lines, lower and upper limits of agreement (LoA). Left (A-C), Main analysis comparing value of record 1 (M2) versus mean value of records 1 , 2, and 3 (M1). Right (D-F), Sensitivity analysis comparing value record 1 (M2) versus mean value of records 2 and 3 (M1).

within the assessor team may be considered to improve consistency of measurements. Another limitation was that in $30 \%$ of all patients pain of electrical stimulation became intolerable before a reflex was evoked, and we performed a complete case analysis in those patients with NWRT. To address this limitation, we compared patients' characteristics of those patients with and without NWRT and found no differences except for age and daily intake of any pain medicaments. We again argue that the magnitude of measurement error is unlikely to be influenced by missing data. A major strength of the presented study is the comprehensive statistical analysis performed to evaluate measurement error. ${ }^{16-18}$ To account for high arithmetic correlation between the mean value of records 1,2 , and 3 and the value of record 1 , a sensitivity analysis was added to compare the value of record 1 with the mean value of records 2 and 3 . Results were much the same as in the main analysis, and thus an underestimation of measurement error due to high arithmetic correlation is unlikely. 
TABLE 3. Results of the Stratified Sensitivity Method Comparison Analysis Showing Between- and Within-Subject SD and ICC Based on Mixed-Effects Linear Regression Models and Systematic Error (SE) and Limits of Agreement (LoA) Based on Bland-Altman Analysis

\begin{tabular}{|c|c|c|c|c|c|c|c|}
\hline & $\mathbf{n}$ & $\begin{array}{l}\text { SD Between } \\
(95 \% \mathrm{CI})\end{array}$ & $\begin{array}{l}\text { SD Within } \\
(95 \% \text { CI) }\end{array}$ & ICC (95\% CI) & SE* $(95 \%$ CI $)$ & $\begin{array}{l}\text { Upper LoA } \dagger \\
(95 \% \text { CI) }\end{array}$ & $\begin{array}{l}\text { Lower LoA } \dagger \\
(95 \% \text { CI) }\end{array}$ \\
\hline \multicolumn{8}{|l|}{ Assessor 1} \\
\hline $\begin{array}{r}\text { PPDT } \ddagger \\
(\mathrm{kPa})\end{array}$ & 101 & $137(119,158)$ & $34(30,39)$ & $0.94(0.92,0.96)$ & $13.57(4.45,22.69)$ & $105.97(90.25,121.70)$ & $-78.83(-94.55,-63.12)$ \\
\hline $\begin{array}{r}\text { EPDT§ } \\
(\mathrm{mA})\end{array}$ & 101 & $3.78(3283,4.34)$ & $0.58(0.51,0.67)$ & $0.98(0.97,0.98)$ & $-0.21(-0.35,-0.06)$ & $1.27(1.02,1.52)$ & $-1.68(-1.93,-1.43)$ \\
\hline $\begin{array}{c}\mathrm{NWRT} \| \\
(\mathrm{mA})\end{array}$ & 63 & $3.09(2.57,3.71)$ & $0.94(0.79,1.12)$ & $0.92(0.87,0.95)$ & $-0.47(-0.77,-0.16)$ & $1.95(1.43,2.47)$ & $-2.88(-3.40,-2.36)$ \\
\hline \multicolumn{8}{|l|}{ Assessor 2} \\
\hline $\begin{array}{c}\text { PPDT, } \$ \\
\mathrm{kPa}\end{array}$ & 88 & $108(93,125)$ & $16(14,19)$ & $0.98(0.97,0.99)$ & $-0.29(-5.19,4.61)$ & $45.97(37.53,54.40)$ & $-46.55(-54.98,-38.12)$ \\
\hline $\begin{array}{l}\text { EPDT, } \S \\
\text { mA }\end{array}$ & 88 & $3.06(2.63,3.56)$ & $0.64(0.55,0.74)$ & $0.96(0.93,0.97)$ & $-0.49(-0.64,-0.34)$ & $0.92(0.67,1.18)$ & $-1.90(-2.16,-1.64)$ \\
\hline $\begin{array}{c}\text { NWRT,\| } \\
\text { mA }\end{array}$ & 65 & $4.08(3.42,4.86)$ & $0.77(0.65,0.91)$ & $0.97(0.94,0.98)$ & $0.07(-0.19,0.33)$ & $2.19(1.74,2.64)$ & $-2.05(-2.50,-1.60)$ \\
\hline \multicolumn{8}{|l|}{ Assessor 3} \\
\hline $\begin{array}{c}\text { PPDT, } \$ \\
\mathrm{kPa}\end{array}$ & 155 & $110(99,124)$ & $17(15,19)$ & $0.98(0.97,0.98)$ & $-0.91(-4.84,3.03)$ & $48.68(41.87,55.49)$ & $-50.50(-57.31,-43.69)$ \\
\hline $\begin{array}{l}\text { EPDT, } \S \\
\mathrm{mA}\end{array}$ & 155 & $3.11(2.78,3.48)$ & $0.50(0.46,0.56)$ & $0.97(0.96,0.98)$ & $-0.05(-0.14,0.05)$ & $1.17(1.00,1.34)$ & $-1.26(-1.43,-1.10)$ \\
\hline $\begin{array}{c}\text { NWRT,\| } \\
\text { mA }\end{array}$ & 114 & $3.94(3.45,4.50)$ & $0.90(0.79,1.02)$ & $0.95(0.93,0.97)$ & $0.08(-0.16,0.32)$ & $2.65(2.24,3.06)$ & $-2.49(-2.90,-2.07)$ \\
\hline \multicolumn{8}{|l|}{ Assessor 4} \\
\hline $\begin{array}{c}\mathrm{PPDT}, \$ \\
\mathrm{kPa}\end{array}$ & 85 & $104(89,122)$ & $26(23,31)$ & $0.94(0.91,0.96)$ & $16.01(8.72,23.30)$ & $83.58(71.04,96.11)$ & $-51.56(-64.10,-39.03)$ \\
\hline $\begin{array}{l}\text { EPDT, } § \\
\mathrm{~mA}\end{array}$ & 85 & $2.91(2.50,3.40)$ & $0.69(0.59,0.80)$ & $0.95(0.92,0.97)$ & $-0.36(-0.53,-0.18)$ & $1.28(0.98,1.59)$ & $-2.00(-2.30,-1.69)$ \\
\hline $\begin{array}{l}\text { NWRT, } \| \\
\text { mA }\end{array}$ & 57 & $6.40(5.30,7.72)$ & $1.35(1.12,1.62)$ & $0.96(0.93,0.97)$ & $-0.30(-0.80,0.20)$ & $3.47(2.61,4.32)$ & $-4.07(-4.92,-3.21)$ \\
\hline \multicolumn{8}{|c|}{$\begin{array}{l}\text { Comparison of the value of record } 1 \text { (M2) with the mean value of records 1, 2, and } 3 \text { (M1) stratified per assessor. All values are presented with corre- } \\
\text { sponding } 95 \% \text { CIs. }\end{array}$} \\
\hline \multicolumn{8}{|c|}{ *SE that corresponds to mean difference between M1 and M2. } \\
\hline \multicolumn{8}{|c|}{$\dagger$ Lower and upper limits of agreement that correspond to SE \pm 1.96 SDs of the SE. } \\
\hline \multicolumn{8}{|c|}{$\$$ ¿PDT at the second toe. } \\
\hline \multicolumn{8}{|c|}{$\S$ EPDT after single electrical stimulation. } \\
\hline \|NWRT a & after s & ingle electrical stim & nulation. & & & & \\
\hline
\end{tabular}

While QSTs are well established in research, their use for clinical practice is not widespread. One reason is the limited data on the ability of QST to support decision making. On the other hand, recent research has shown potential prognostic value and ability to predict efficacy of medications, although the results are not consistent across studies studies. ${ }^{23-25}$ Importantly, detecting central sensitization with QST may help patients better understand their pain condition. Simplifying the testing procedure is expected to lead to broader use in clinical practice and hopefully to more large-scale studies that provide insights on the role of QST in the clinical management of pain.

To ensure comparability of research results and to facilitate wide use in clinical practice, an evidence-based approach toward standardization of QST assessment procedures is needed. This study contributes to a standardized QST testing protocol. One single record of PPDT, EPDT, and NWRT is associated with minimal measurement error, compared with the mean of 3 sequential records. Based on this result, it seems acceptable to limit the number of records to 1 . We expect to save time, resources, and patients' discomfort when reducing the number of records to 1 .

\section{REFERENCES}

1. Woolf CJ. Central sensitization: implications for the diagnosis and treatment of pain. Pain. 2011;152:S2-S15.

2. Curatolo M. Diagnosis of altered central pain processing. Spine (Phila Pa 1976). 2011;36:S200-S2004.

3. Birklein F, Sommer C. Pain: quantitative sensory testing - a tool for daily practice? Nat Rev Neurol. 2013;9:490-492.

4. O'Neill S, Manniche C, Graven-Nielsen T, Arendt-Nielsen L. Generalized deep-tissue hyperalgesia in patients with chronic low-back pain. Eur J Pain. 2007; 11:415-420

5. Sterling M. Differential development of sensory hypersensitivity and a measure of spinal cord hyperexcitability following whiplash injury. Pain. 2010;150:501-506.

6. Backonja MM, Attal N, Baron R, et al. Value of quantitative sensory testing in neurological and pain disorders: NeuPSIG consensus. Pain. 2013;154:1807-1819

7. Rolke R, Baron R, Maier C, et al. Quantitative sensory testing in the German research network on neuropathic pain (DFNS): standardized protocol and reference values. Pain. 2006;123:231-243. 
8. Curatolo M, Muller M, Ashraf A, et al. Pain hypersensitivity and spinal nociceptive hypersensitivity in chronic pain: prevalence and associated factors. Pain. 2015;156:2373-2382.

9. World Medical Association. World Medical Association Declaration of Helsinki. Ethical principles for medical research involving human subjects. JAMA. 2013;310:2191-2194.

10. Poole H, Bramwell R, Murphy P. The utility of the Beck Depression Inventory Fast Screen (BDI-FS) in a pain clinic population. Eur J Pain. 2009; 13:865-869.

11. Rosenstiel AK, Keefe FJ. The use of coping strategies in chronic low back pain patients: relationship to patient characteristics and current adjustment. Pain. 1983;17:33-44.

12. Neziri AY, Curatolo M, Limacher A, et al. Ranking of parameters of pain hypersensitivity according to their discriminative ability in chronic low back pain. Pain. 2012;153:2083-2091.

13. Brennum J, Kjeldsen M, Jensen K, Jensen TS. Measurements of human pressure-pain thresholds on fingers and toes. Pain. 1989;38:211-217.

14. Willer JC. Comparative study of perceived pain and nociceptive flexion reflex in man. Pain. 1977;3:69-80.

15. Rhudy JL, France CR. Reliability and validity of a brief method to assess nociceptive flexion reflex (NFR) threshold. J Pain. 2011;12:782-791.

16. Bartlett JW, Frost C. Reliability, repeatability and reproducibility: analysis of measurement errors in continuous variables. Ultrasound Obstet Gynecol. 2008;31:466-475

17. Bland JM, Altman DG. Statistical methods for assessing agreement between two methods of clinical measurement. Lancet. 1986;1:307-310.
18. Altman DG, Bland JM. Measurement in medicine: the analysis of method comparison studies. The Statistician. 1983;32:307-317.

19. Shrout PE, Fleiss JL. Intraclass correlations: uses in assessing rater reliability. Psychol Bull. 1979;86:420-428.

20. Biurrun Manresa JA, Neziri AY, Curatolo M, Arendt-Nielsen L, Andersen OK. Test-retest reliability of the nociceptive withdrawal reflex and electrical pain thresholds after single and repeated stimulation in patients with chronic low back pain. Eur J Appl Physiol. 2011;111: $83-92$.

21. Geber C, Klein T, Azad S, et al. Test-retest and interobserver reliability of quantitative sensory testing according to the protocol of the German research network on neuropathic pain (DFNS): a multi-centre study. Pain. 2011;152:548-556.

22. Vuilleumier PH, Biurrun Manresa JA, et al. Reliability of quantitative sensory tests in a low back pain population. Reg Anesth Pain Med. 2015;40: 665-673.

23. Sterling M, Jull G, Kenardy J. Physical and psychological factors maintain long-term predictive capacity post-whiplash injury. Pain. 2006;122: $102-108$

24. Mainka T, Malewicz NM, Baron R, Enax-Krumova EK, Treede RD, Maier C. Presence of hyperalgesia predicts analgesic efficacy of topically applied capsaicin $8 \%$ in patients with peripheral neuropathic pain. Eur J Pain. 2016;20:116-129.

25. Mlekusch S, Schliessbach J, Cámara RJ, Arendt-Nielsen L, Jüni P, Curatolo M. Do central hypersensitivity and altered pain modulation predict the course of chronic low back and neck pain? Clin J Pain. 2013;29:673-680. 